\title{
Multiple Removal by Wavepath Migration
}

\author{
Yike Liu ${ }^{*} \quad \mathrm{Xu}$ Chang ${ }^{*}$ Hongchuan Sun ${ }^{* *}$ \\ * Institute of Geology and geophysics, Chinese Academy of Sciences, Beijing 100029 \\ ** The department of Geology and Geophysics, University of Utah
}

\begin{abstract}
Multiple removal by wavepath migration is used for discriminating primary reflections from multiple reflections during the migration process. Both the take-off and incidence angles are computed from the data so that rays can be traced from both the source and the receiver positions. An event is a primary reflections as long as the rays intersect and the intersection point has a reflection traveltime equal to the observed traveltime. In contrast, an event is a multiple reflection if the intersection point of the rays has a reflection traveltime different from he observed traveltime. Numerical results for a three-layer model show that multiple removal by wavepath migration can clearly resolve the true interfaces and effectively remove the false structures generated by multiple energy. Numerical results show that the method can effectively remove the migration artifacts generated by surface-related multiples. When near-offset data are not used, the migrated images is nearly free of multiple-related artifacts. However, the method becomes less effective with decreasing source-receiver offset of the traces.
\end{abstract}

Key Words: seismic migration, demultiple, wavepath

\section{Introduction}

It is a challenging task to attenuate and remove multiple from seismic data. The suppression of multiple event is a crucial task when processing and explaining to offshore seismic data even as seismic static correction is a key factor to onshore seismic data (Liu, 2001, Chang 2001). There are many conventional and newly-developed techniques that aim to attenuate multiples. Filtering in the $f-k$ or the tau-p domains uses the separability of primary and multiple reflections in the transformed domain due to their differences in 
moveout. The predictive methods, such as the predictive deconvolution method and the model and data-based prediction and subtraction methods, use the predictability of the multiples from the primary events. However, in the deep-water case where the length of the available record is not long enough compared to the reverberation period, poor autocorrelation estimates result in the predictive deconvolution not working. The wave-equation-based methods are developed for eliminating multiple $e^{[5-8]}$, the effectiveness of the method depends upon the multiple modeling and subtraction factor. Although inversion method exists ill-posed problems, it is exploited in eliminating multiple ${ }^{[1]}$.(Luo Yi). Inversion method to attenuate multiples proved (Wang, Sun and Schuster) present a migration algorithm denoted as wavepath migration, The migration method give rise to fewer migration artifacts and can define complex structure better than Kirchhoff migration. In this paper, we gave a multiple removal method in the basis of the wavepath migration.

\section{Theory}

Seismic data consist of both primary and multiple events reflected at subsurface discontinuities. A primary event is only reflected once at a layer interface while a multiple event is reflected more than once at different interfaces. Figure 1a illustrates the ray path of a primary reflection, where seismic energy propagates down into the earth at some take-off angle, is reflected at an interface, and finally returns to the surface at some incidence angle. Similarly, a multiple reflection has its own take-off and incidence angles, but these angles are usually different from those of a primary reflection. Figure $1 \mathrm{~b}$ shows a kind of surface-related multiple, whose energy is reflected several times before it returns to the receiver. 


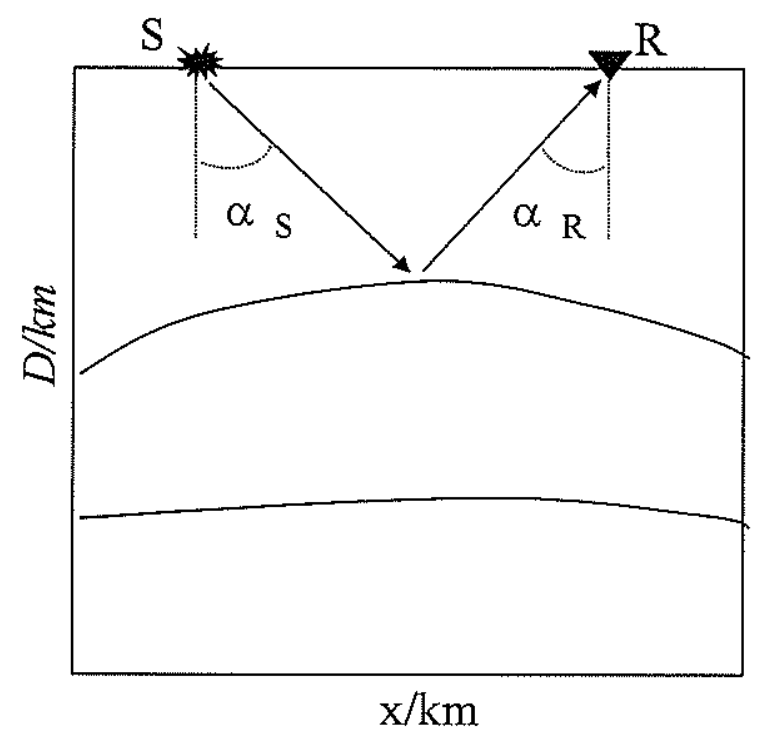

Fig. 1(a)

Fig.1 (a) A primary reflection is only reflected at one interface;(b) a multiple reflection is reflected more than once at different interfaces.

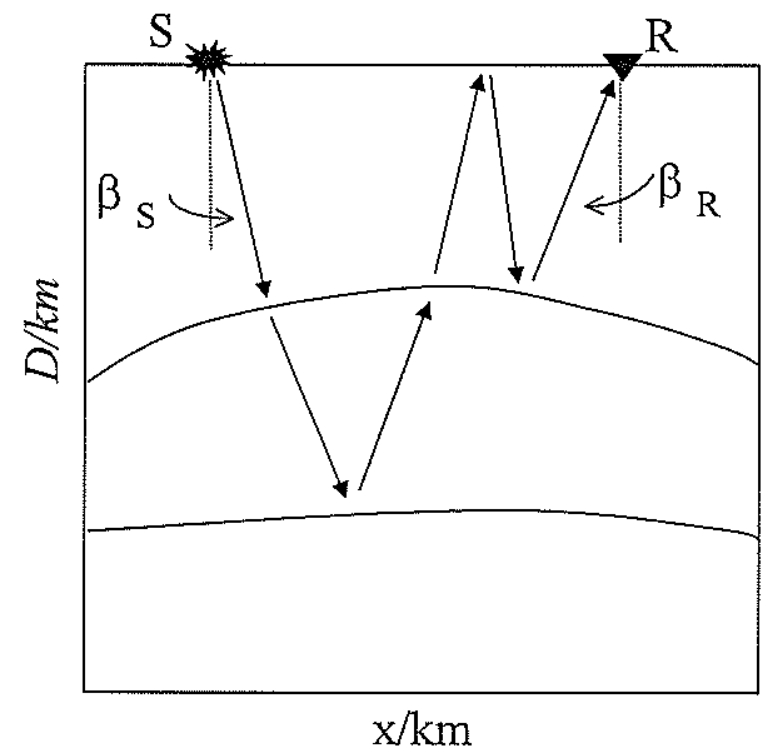

Fig.1 (b)

Fig. 2 (a) never intersect, or (b) finally intersect, but the intersection point is not at the correct reflector position 


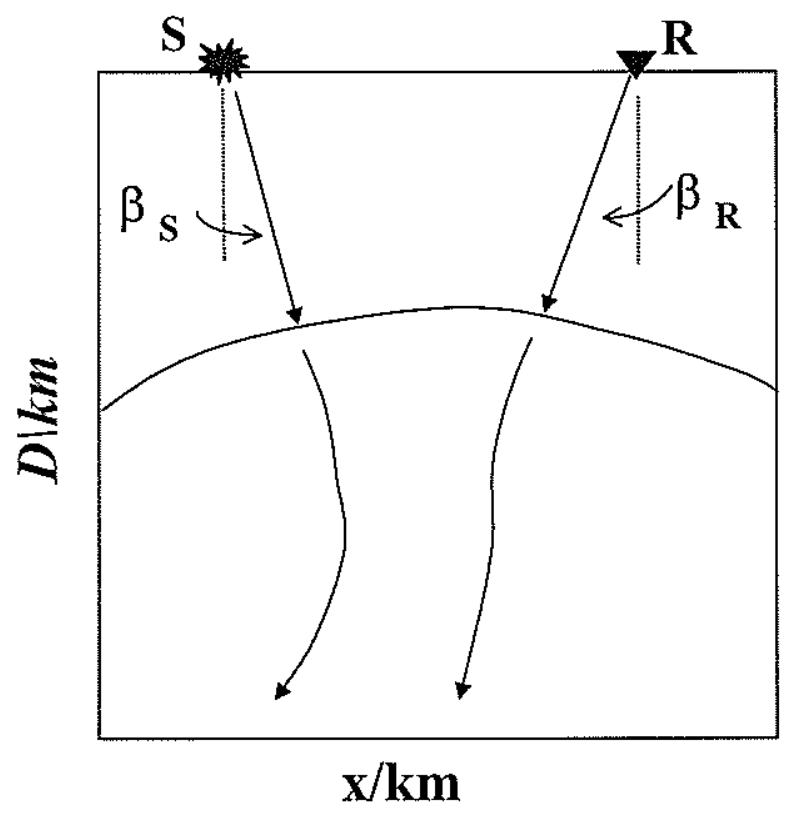

Fig2(a)

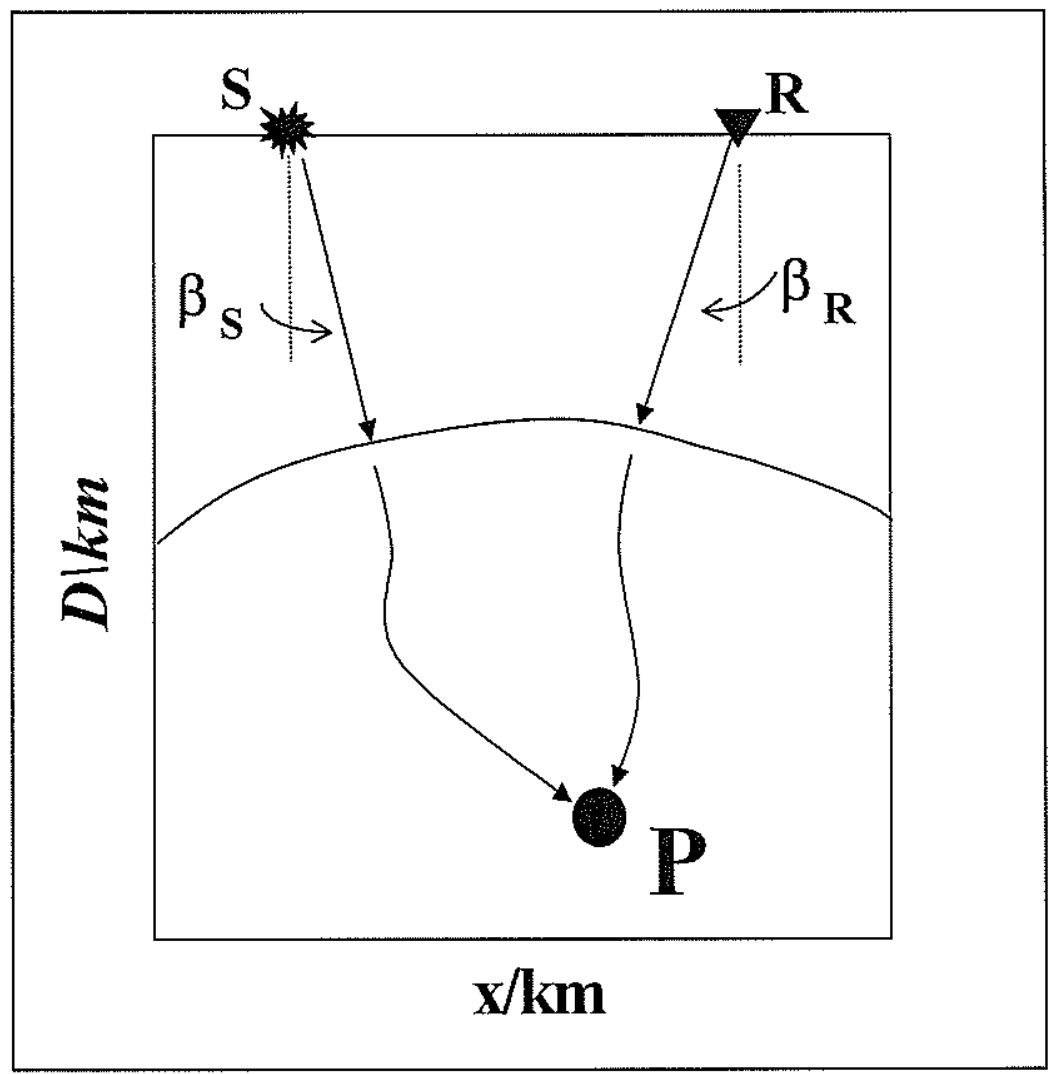

Fig. 2 show how to migrate a primary reflection with wavepath migration。In migration, 
the travel time imaging condition is described by the following equation:

$$
\tau_{S R}^{o b s}=\tau_{S P}^{c a l}+\tau_{R P}^{c a l}
$$

where $\mathrm{S}, \mathrm{R}$ and $\mathrm{P}$ denote the locations of the source, receiver and two-ray crossing point, respectively; $\tau_{S R}^{o b s}$ is the observed reflection traveltime, $\tau_{S P}^{c a l}$ is the calculated traveltime from the source to the two-ray crossing point $\mathrm{P}$, and $\tau_{R P}^{c a l}$ is the calculated traveltime from the receiver to point $P$.

Like primary reflection migration, one ray is traced from the source position with shooting angle $\beta_{s}$, and another ray is traced from the receiver position with shooting angle $\beta_{R}$. Unlike primary reflection migration, here the two rays may might finally intersection, but the two-ray crossing point $P$ has a calculated reflection traveltime $\tau_{S P}^{c a l}+\tau_{R P}^{c a l}$ not equal to the observed traveltime $\tau_{S R}^{o b s}$. For either case, the event is regarded as a multiple reflection and will not be migrated.

(1) both the take-off and incidence angles are estimated by performing a local slant stack over a window of traces centered about the picked event. The take-off angle is estimated in a common receiver gather, whereas the incidence angle is estimated in a common shot gather.

(2) One ray is traced from the receiver position with a shooting angle equal to $\alpha_{R}$. Along the raypath, a specular reflection point $\mathrm{P}$ is determined.

(3) Another ray is traced from the source position with a shooting angle equal to $\alpha_{S}$, Along the raypath, another specular reflection point $\mathrm{Q}$ is determined for the same picked event.

(4) If the image points $P$ and $Q$ are far away from each other, then the event is regarded as a multiple reflection and not further processing is carried out. However, of points $\mathrm{P}$ and $\mathrm{Q}$ coincide and the associated traveltime is equal to the observed traveltime, then the event is regarded as a primary reflection and its energy is smeared along the Fresnel zone centered about the image point $P$. 
(5) In practice, the image points $P$ and $Q$ hardly coincide, and the traveltime can hardly be equal to. As a result, the requirements are relaxed to be:

$$
\|P-Q\| \leq \delta x
$$

(2)

$$
\left\|\tau_{S R}^{o b s}-\tau_{S Q}^{c a l}-\tau_{R P}^{c a l}\right\| \leq \delta \tau
$$

$\delta x$ and $\delta \tau$ are tolearable spatial and traveltime errors, respectively.

\section{Numerical Calculation}

3. 1 Three-Layer Model

The model consists of three horizontal layers, and is discretized into a $401 * 1000$ grid, with a horizontal and vertical $5 \mathrm{~m}$. There are 81 sources and 81 receivers uniformly distributed on the surface, with source and receiver internals equal to $25 \mathrm{~m}$. Figure $3 \mathrm{~b}$ shows one of the synthetic shot gathers, which were generated by using a finite-difference methos with a source frequency of $25 \mathrm{~Hz}$. Figure $3 \mathrm{c}$ shows the Kirchhoff migration image, where the true interfaces are clearly resolved, but the multiple energy is very strong and gives rise to false structures, Figure $3 \mathrm{~d}$ shows the wavepath migration with the multiple removal by wavepath migration, where the multiple energy is significantly attenuated.

(a)

(b) 


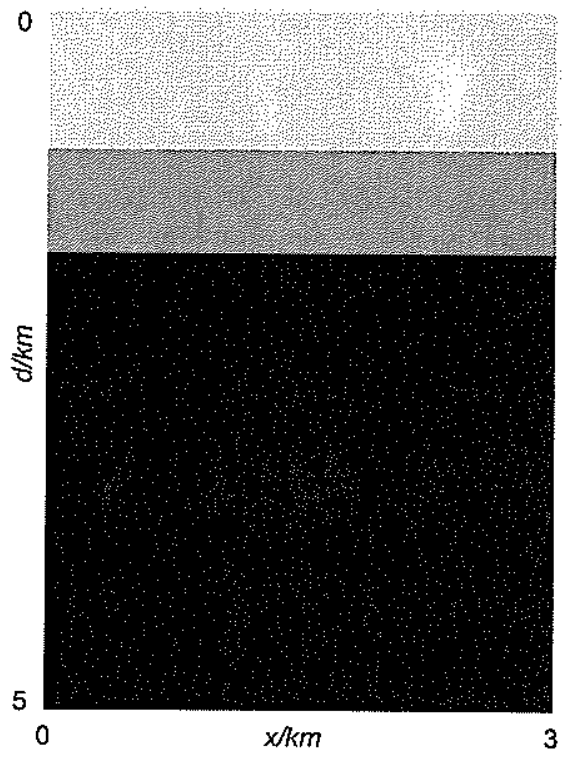

(c)

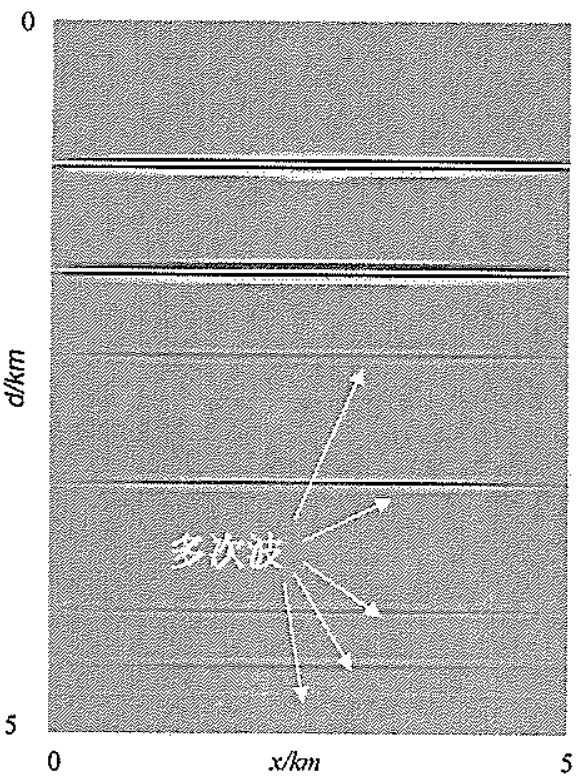

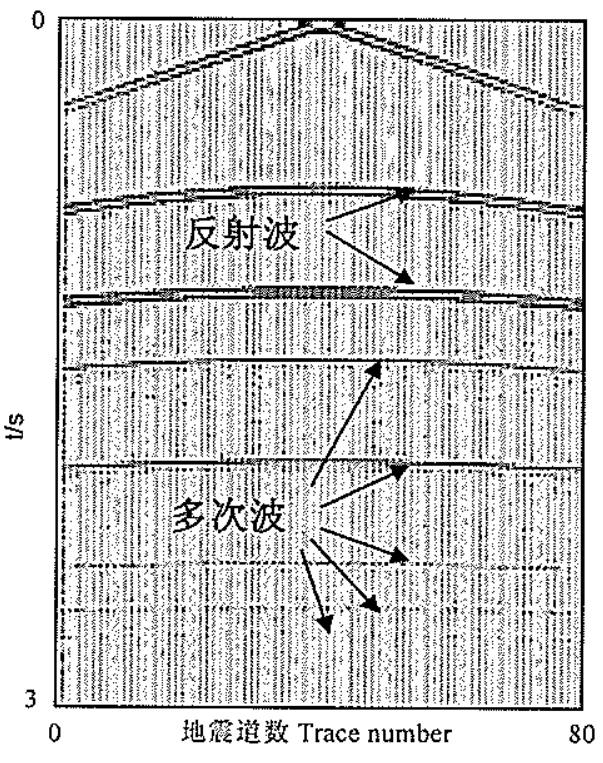

(d)

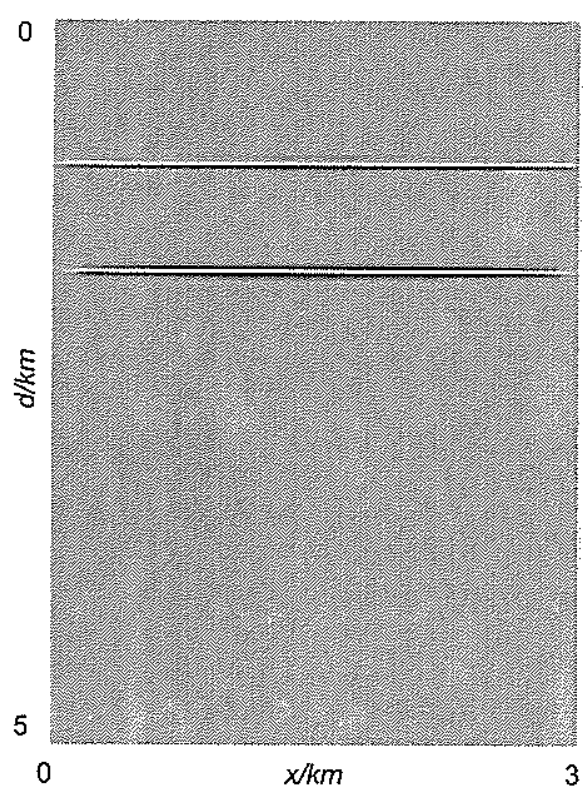

Figure3 A 3-layer velocity model

(a) velocity model; (b) one shot gather seismograms; (c) Kirchhoff image; (d) wavepath migration image by demultiple. 


\section{2 Five-Layer velocity model}

For a five-layer model with a lateral velocity change, a finite-difference method is used to generate data for 121 receivers and 121 sources on the surface with receiver and source intervals equal to $25 \mathrm{~m}$. The four primary reflections are denoted in Figure $4 \mathrm{~b}$ by $\mathrm{P} 1 、 \mathrm{P} 2 、 \mathrm{P} 3$ and $\mathrm{P} 4$, respectively. The $\mathrm{P} 3$ primary reflection is nearly coincident with a multiple-reflection event at the near offset location.Figure $4 \mathrm{c}$ shows the Kirchhoff migration image. The multiple energy is very strong and generates an artifical multiple image. The true layer 2 image overlaps the artifical multiple image just below it. Figure $4 \mathrm{~d}$ shows the migration image after the primary only image condition migration is applied. The multiple energy is significantly attenuated. Because the primary reflections from layer 4 are very weak and the incidence angles are not calculated vey accurately, the image for this layer is weaker after applying the primary only image condition.

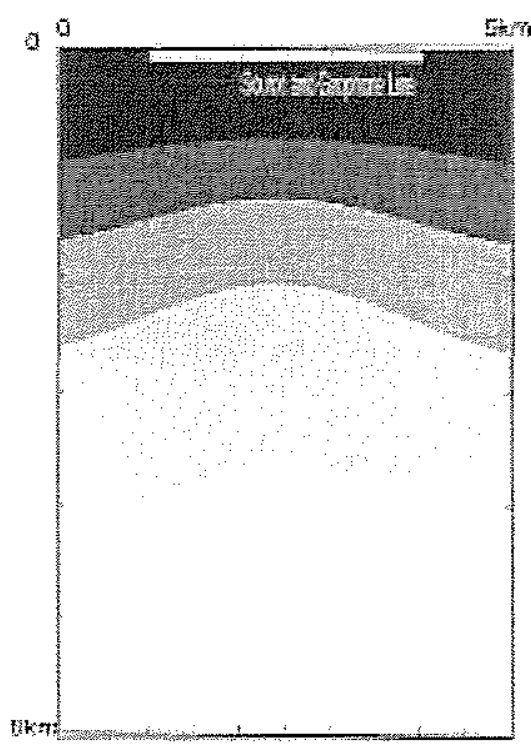

iat

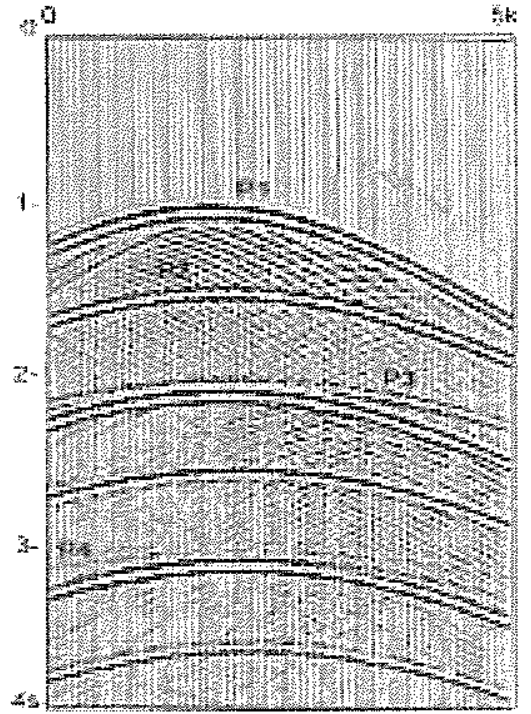

(b) 

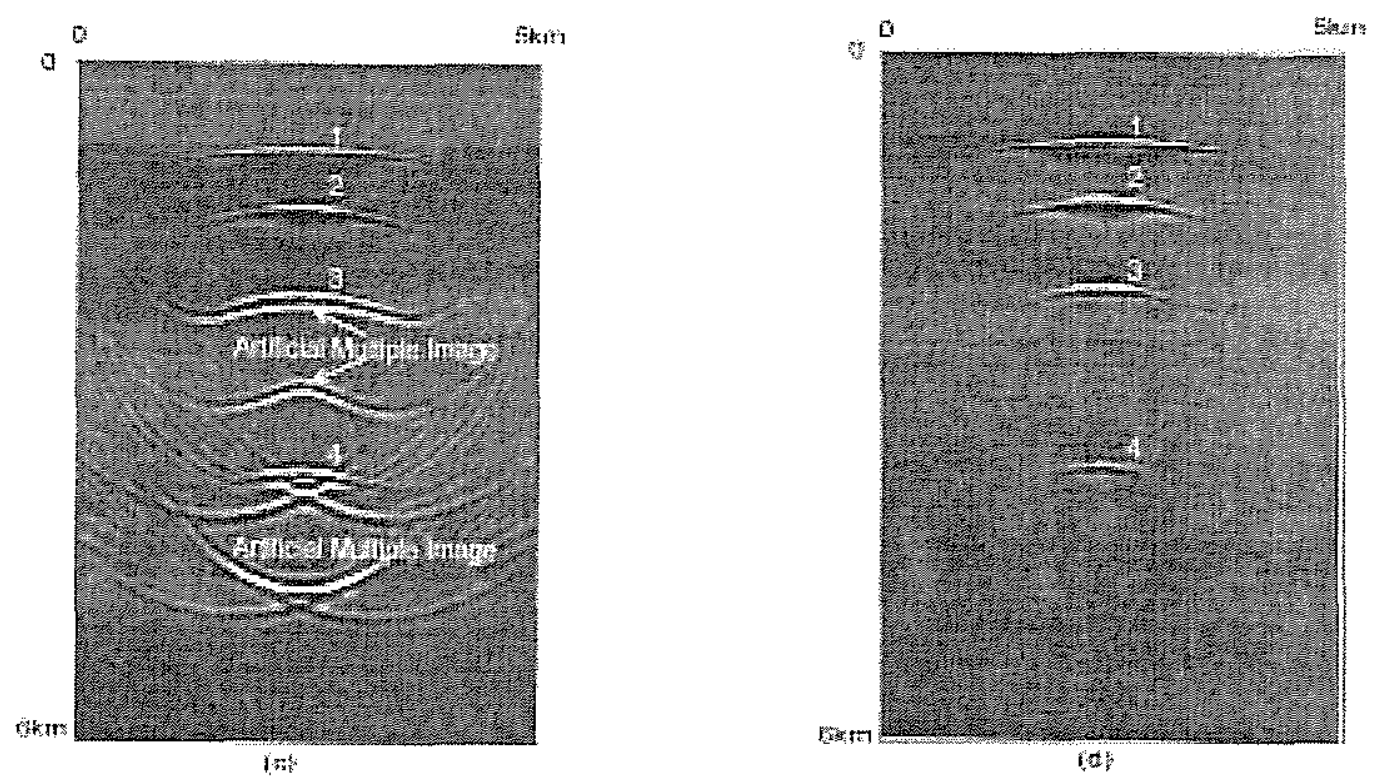

Figure4 5-layercurve velocity model

(a) velocity model; (b) one shot gather seismograms; (c) Kirchhoff image; (d) wavepath migration image by demultiple.

\section{Conclusion}

Multiple removal by wavepath migration remove multiple form seismic data and does not need to know the periodicity, amplitude and phase. It removes multiple during the migration based on the behavior of primary and multiple by calculating the ray path through imaging point, Fresnel zone, Green function, spreading factor and migration aperture. It can attenuate the surface related and interval multiples and suppresion the artifacts. The results of migration illustrate the methods is effective to lateral velocity change model. There still exist difficult in separating primary and multiple in near offset and the method is a little sensitive to velocity model.

The research was funded by the national Natural Sciences Foundation of China (grant No. 
40374050, 40235055), and National High Technology Research and Development Program of China (863 Program). We thank Gerard T. Schuster and Yue Wang from University of Utah, who provided helpful and insightful comments.

\section{References}

[1] Liu, Yike, Chang, Xu, Wang Hui. Estimation of near-surface velocity for 3-D complicated topography and seismic tomographic static corrections. Chinese Journal of Geophysics, 2001, 44 (2) 268 274.

[2] Chang, X, Liu, Y, Wang, H, Li, F, and Chen, J. 3-D tomographic static correction. Geophysics, 2001, 67(4), $1275 \sim 1285$.

[3] Hampson, D. Inverse velocity stacking for multiple elimination: J. Can., Soc, Expl. Geophys., 1988, 22(1), $49 \sim 55$.

[4]Foster, D. J, and Mosher, C. C. suppression of multiple reflection using the Radon transform: Geophysics, 1992, 57(3), 386 395.

[5] Berkhout, A. J, and Verschuur, D. J. Estimation of multiple scattering by iterative inversion, part 1-Theoretical considerations: Geophysics, 1997, 62(5), $1586 \sim 1595$.

[6] Wiggins, R. A, and Robinson, E. A. Recursive solution of the multichannel filtering problem. J. Geophys. Res., 1965, 70, $1885 \sim 1891$.

[7] Verschuur, D. J, and Berkhout, A. J. Estimation of multiple scattering by iterative inversion, part II-Practical aspects and examples: Geophysics, 1997, 62(5), $1596 \sim 1611$.

[8] Zhou B, Greenhalgh S A. Wave equation extrapolation-based multiple attenuation: 2D filter in the F-K domain, Geophysics, 1994, 59(9), 1377 1391.

[9]Chang Xu, Liu,Yike. The Improvement of Precision of the Lateral Estimation in the Target Strata Using the Velocity Tomographic Technique. Chinese Journal of Geophysics, 1997, 40(1).127 137.

[10] Liu, Yike, Chang, Xu. Quatitative assessment of inversion solution of seismic tomography and its application. Chinese Journal of Geophysics, 2000, .43(2),

[11] Wang, Y. H. Multiple subtraction using an expanded multichannel matching filter: Geophysics, 2003, 68(1), 346 354.

[12] Wang, $Y$, Sun, H, and Schuster, G. T. Migration image condition for primary 
reflections: $69^{\text {th }}$ Ann. Internat. Mtg. Soc. Expl. Geophys., Expanded Abstracts, 1999, $1126 \sim 1129$.

[13] Sun, H, Schuster, G. T. 2-D Wavepath Migration. Geophysics, 2001, 67(5), 1528 1537. 